\title{
Studies of Nonlinear Sound Dynamics in Fluids Based on the Caloric Equation of State
}

\author{
Anna PERELOMOVA, Paweł WOJDA \\ Gdańsk University of Technology \\ Faculty of Applied Physics and Mathematics \\ Narutowicza 11/12, 80-233 Gdańsk, Poland \\ e-mail: anpe@mif.pg.gda.pl, pwojda@mif.pg.gda.pl \\ (received June 25, 2009; accepted October 6, 2010)
}

\begin{abstract}
The sound speed and parameters of nonlinearity $B / A, C / A$ in a fluid are expressed in terms of coefficients in the Taylor series expansion of an excess internal energy, in powers of excess pressure and density. That allows to conclude about features of the sound propagation in fluids, the internal energy of which is known as a function of pressure and density. The sound speed and parameters of nonlinearity in the mixture consisting of boiling water and its vapor under different temperatures, are evaluated as functions of mass concentration of the vapor. The relations analogous to that in the Riemann wave in an ideal gas are obtained in a fluid obeying an arbitrary equation of state. An example concerns the van der Waals gases. An excess pressure in the reflected wave, which appears when standard or nonlinear absorption in a fluid takes place, is evaluated in an arbitrary fluid.
\end{abstract}

Keywords: nonlinear acoustics, parameters of nonlinearity, equations of state, Riemann wave.

PACS No. 43.25, 43.25.Jh

\section{Introduction}

The nonlinearity parameters $B / A$ and $C / A$ play a significant role in nonlinear acoustics, in a number of areas ranging from underwater acoustics to medicine (Novikov et al., 1988; Carstensen, Bacon, 1998). The ratios $B / A$ and $C / A$ have their origins in the Taylor series of the expansion of the variations of the pressure in a fluid, in terms of variations of the density. The changes are carried out reversibly, adiabatically and at constant chemical composition:

$$
\begin{aligned}
p^{\prime}=p-p_{0}=\left(\frac{\partial p^{\prime}}{\partial \rho}\right)_{s, 0} \rho^{\prime}+ & \frac{1}{2}\left(\frac{\partial^{2} p^{\prime}}{\partial \rho^{2}}\right)_{s, 0} \rho^{\prime 2}+\frac{1}{6}\left(\frac{\partial^{3} p^{\prime}}{\partial \rho^{3}}\right)_{s, 0} \rho^{\prime 3}+\ldots \\
& =A\left(\frac{\rho^{\prime}}{\rho_{0}}\right)+\frac{B}{2}\left(\frac{\rho^{\prime}}{\rho_{0}}\right)^{2}+\frac{C}{6}\left(\frac{\rho^{\prime}}{\rho_{0}}\right)^{3}+\ldots
\end{aligned}
$$


where $p, p_{0}$ denote pressure and its ambient value, $\rho, \rho_{0}$ denote density and its ambient value, $p^{\prime}=p-p_{0}$ is the sound pressure, and $\rho^{\prime}=\rho-\rho_{0}$ is an excess fluid density. In nonlinear acoustics, the current notation was perhaps first employed by these authors (FOX, WALLACE, 1954). The isentropic small-signal sound speed is defined by the following equality:

$$
c_{0}^{2}=\left(\frac{\partial p^{\prime}}{\partial \rho}\right)_{s, 0}=\frac{A}{\rho_{0}} .
$$

The partial derivatives in Eqs. (1), (2) are all evaluated at the unperturbed state $\left(\rho_{0}, s_{0}\right)$, what is indicated by the subscript 0 . Parameters of nonlinearity in fluids may be obtained from the variation of sound velocity with temperature and pressure (BEYER, 1998):

$$
\begin{aligned}
& \frac{B}{A}=2 \rho_{0} c_{0}\left(\frac{\partial c}{\partial p}\right)_{s, 0}=2 \rho_{0} c_{0}\left(\frac{\partial c}{\partial p}\right)_{T, 0}+2 \frac{\alpha_{T} c_{0} T_{0}}{C_{p}}\left(\frac{\partial c}{\partial T}\right)_{p, 0}, \\
& \frac{C}{A}=\frac{3}{2}\left(\frac{B}{A}\right)^{2}+2 \rho_{0}^{2} c_{0}^{3}\left(\frac{\partial^{2} c}{\partial p^{2}}\right)_{s, 0},
\end{aligned}
$$

where $\alpha_{T}=\rho_{0}\left(\partial \rho^{-1} / \partial T\right)_{p_{0}}$ is the volumetric coefficient of thermal expansion, $C_{P}$ is specific heat at constant pressure, $T_{0}$ is the ambient temperature.

Evaluation of the ratios $B / A, C / A$ obviously requires precise knowledge about function $c(p, T)$ on extensive area of equilibrium states. Usually these data are established experimentally. Alternative, but a more convenient way, is to express sound speed and parameters of nonlinearity in terms of series of an excess internal energy $e(\rho, T)$, since the recent advances in physical chemistry allow to approximate internal energy for most models of forces between molecules (based on atomic model of hard spheres, soft spheres and other) with reasonably high accuracy. That is of great importance for liquids, even for such anomalous like water (LeE et al., 1963; Jeffrey, Austin, 1999). Knowledge about internal forces between molecules in a fluid provides a fairly high accuracy in calculating of thermodynamic potentials like free energy and therefore, an internal energy of a fluid. Vice versa, acoustic measurements of a waveform nonlinear distortion may correct conclusions of chemical physics (SEHGAL, 1995). As the zero-order test, analytically derived thermodynamic potentials must provide a correct value of the sound speed. Comparison of theoretical predictions of $B / A$ and $C / A$ with their experimental values would allow to correct a curvature of the surface representing internal energy as a function of $\rho, T$. Sections 1, 2 are devoted to estimation of the small-signal sound speed and parameters of nonlinearity in the media, where internal energy as a function of density and temperature is known analytically, like mixture consisting of boiling water and its vapor, or van der Waals gases. The final formulae include coefficients in the Taylor series expansion of an excess internal energy $e(\rho, T)$. 
Analytical method is much more convenient in many applications for one more reason. The conservative equations of mass, momentum and energy are similar in gases and liquids. The sound speed and parameter of nonlinearity $B / A$ are larger in liquids (BEYER, 1960; 1998; COPPENS et al., 1965). The reason for that is a difference in thermodynamic properties of gases and liquids. Thermodynamic properties follow in fact from two equations of state, the caloric and thermal ones, the first one connecting internal energy of a medium with two thermodynamic forces, pressure and temperature, and the second one connecting density with pressure and temperature. Dynamics of any fluid may be considered by means of the Taylor series expansion of both equations of state. That permits to draw general conclusions about nonlinear features of any fluid motion and, in particular, about nonlinear features of propagation of sound itself.

The determination of nonlinear waveforms like the one-dimensional Riemann wave in ideal gases (but in fluids different from an ideal gas) is also an important problem. The Riemann wave is known as an exclusive example of exact solution of conservative nonlinear equations in the differential form, describing a non-viscous ideal gas (Riemann, 1953; Rudenko, Soluyan, 1977).

It is established by relations linking acoustic pressure, excess density and velocity of a fluid. These nonlinear relationships are known within any accuracy only for an ideal gas motion. In Sec. 1, the links specific for the Riemann wave in any fluid are evaluated within the accuracy up to the third-order nonlinear terms. The way to determine these relations within any accuracy is also pointed out there. The reflected wave excess pressure in any absorbing fluid is derived by use of the relations specific for the Riemann wave in Subsec. 2.2. An example concerns reflected pressure in an ideal gas within the accuracy up to the nonlinear term proportional to the third power of velocity, and the reflected wave in van der Waals gases.

\subsection{Basic system and nonlinear links for sound}

In order to determine the parameters of nonlinearity, it is sufficient to consider one-dimensional flow along axis $O X$ without account of thermoviscous dissipation. Nor dissipation neither a non-planarity of a flow input in parameters of nonlinearity. The parameters $B / A, C / A$ are exclusively functions of the caloric state. The theoretical description of finite-amplitude sound has its origins in the continuity, the momentum, and the energy equations:

$$
\begin{aligned}
\frac{\partial \rho}{\partial t}+\frac{\partial(\rho v)}{\partial x} & =0, \\
\rho\left(\frac{\partial v}{\partial t}+v \frac{\partial \rho}{\partial x}\right)+\frac{\partial p}{\partial x} & =0 \\
\frac{\partial e}{\partial t}+v \frac{\partial e}{\partial x}+\frac{p}{\rho} \frac{\partial v}{\partial x} & =0
\end{aligned}
$$


where $v$ denotes particle velocity of a fluid, $x, t$ are spacial co-ordinate and time, respectively. The system (4) should be completed by the caloric equation of state. This last one specifies thermodynamic and, consequently, acoustic properties of an individual fluid. An excess internal energy $e^{\prime}$ may be represented as a series in powers of excess density and pressure $\rho^{\prime}, p^{\prime}$ in the most general form:

$$
\begin{aligned}
\rho_{0} e^{\prime}=E_{1} p^{\prime}+\frac{E_{2} p_{0}}{\rho_{0}} \rho^{\prime}+\frac{E_{3}}{p_{0}} p^{2}+\frac{E_{4} p_{0}}{\rho_{0}^{2}} \rho^{\prime 2}+\frac{E_{5}}{\rho_{0}} p^{\prime} \rho^{\prime} \\
+\frac{E_{6}}{p_{0} \rho_{0}} p^{\prime 2} \rho^{\prime}+\frac{E_{7}}{\rho_{0}^{2}} p^{\prime} \rho^{\prime 2}+\frac{E_{8}}{p_{0}^{2}} p^{\prime 3}+\frac{E_{9} p_{0}}{\rho_{0}^{3}} \rho^{\prime 3}+\ldots,
\end{aligned}
$$

where $E_{1}, \ldots E_{9}$ are correspondent partial derivatives, all evaluated at the unperturbed state $\left(p_{0}, \rho_{0}\right)$. In the frames of the present study, we maintain terms of power no higher than the third one in the series (5).

In order to establish links analogous to those in the Riemann wave, the waveform has to be simple a priori. This means that any wave perturbation may be expressed in terms of only one referring quantity; for example, velocity and acoustic pressure are functions of an excess density (RIEMANN, 1953; RUDENKO, SOLUYAN, 1977). Within the accepted accuracy, the relationships take the form

$$
\begin{aligned}
& \frac{v^{\prime}(x, t)}{c_{0}}=M_{1} \frac{\rho^{\prime}}{\rho_{0}}+M_{2}\left(\frac{\rho^{\prime}}{\rho_{0}}\right)^{2}+M_{3}\left(\frac{\rho^{\prime}}{\rho_{0}}\right)^{3}, \\
& \frac{p^{\prime}(x, t)}{\rho_{0} c_{0}^{2}}=M_{4} \frac{\rho^{\prime}}{\rho_{0}}+M_{5}\left(\frac{\rho^{\prime}}{\rho_{0}}\right)^{2}+M_{6}\left(\frac{\rho^{\prime}}{\rho_{0}}\right)^{3},
\end{aligned}
$$

where $c_{0}=\sqrt{\frac{\left(1-E_{1}\right) p_{0}}{E_{2} \rho_{0}}}$. The constants $M_{1}, \ldots M_{6}$ are to be determined from the condition that substituting of $p^{\prime}, v^{\prime}$ in terms of $\rho^{\prime}$ in Eqs. (4) produces three equivalent dynamic equations governing an excess density. Equating of linear, quadratic and cubic terms of all three equations yields six algebraic equations determining the unknown quantities $M_{1}, \ldots M_{6}$. After some algebraic manipulations, one gets relations analogous to those in the Riemann wave progressive in the positive direction of axis $O X\left(M_{1}>0\right)$ :

$$
\begin{aligned}
\frac{v^{\prime}(x, t)}{c_{0}}= & \frac{\rho^{\prime}}{\rho_{0}}-\frac{1}{4}\left(3+D_{1}+D_{2}\right)\left(\frac{\rho^{\prime}}{\rho_{0}}\right)^{2} \\
& +\left(\frac{5}{8}+\frac{D_{1}}{3}+\frac{D_{2}}{4}-\frac{D_{3}}{6}-\frac{D_{4}}{6}-\frac{D_{5}}{6}+\frac{D_{1}^{2}}{24}-\frac{D_{2}^{2}}{24}\right)\left(\frac{\rho^{\prime}}{\rho_{0}}\right)^{3}, \\
\frac{p^{\prime}(x, t)}{\rho_{0} c^{2}}= & \frac{\rho^{\prime}}{\rho_{0}}-\frac{1}{2}\left(1+D_{1}+D_{2}\right)\left(\frac{\rho^{\prime}}{\rho_{0}}\right)^{2} \\
& +\left(\frac{1}{3}+\frac{D_{1}}{2}+\frac{D_{2}}{3}-\frac{D_{3}}{3}-\frac{D_{4}}{3}-\frac{D_{5}}{3}+\frac{D_{1}^{2}}{6}-\frac{D_{1} D_{2}}{6}\right)\left(\frac{\rho^{\prime}}{\rho_{0}}\right)^{3} .
\end{aligned}
$$


Constants $D_{1}, \ldots D_{5}$ are as follows:

$$
\begin{aligned}
& D_{1}=\frac{1}{E_{1}}\left(-1+2 \frac{1-E_{2}}{E_{1}} E_{3}+E_{5}\right), \\
& D_{2}=\frac{1}{1-E_{2}}\left(1+E_{2}+2 E_{4}+\frac{1-E_{2}}{E_{1}} E_{5}\right), \\
& \begin{aligned}
& D_{3}= \frac{1}{1-E_{2}}\left(1-3 E_{9}-2 E_{4}-\frac{E_{7}\left(1-E_{2}\right)}{E_{1}}\right. \\
&\left.+\frac{E_{5}}{E_{1}}\left(1+E_{2}+2 E_{4}\right)+\frac{E_{5}^{2}\left(1-E_{2}\right)}{E_{1}^{2}}\right), \\
& D_{4}= \frac{1-E_{2}}{E_{1}^{3}}\left(\frac{4 E_{3}^{2}\left(1-E_{2}\right)}{E_{1}}-E_{1} E_{6}+2 E_{3} E_{5}-3 E_{8}\left(1-E_{2}\right)-2 E_{3}\right), \\
& D_{5}= \frac{1}{E_{1}^{2}}\left(\frac{4 E_{3} E_{5}\left(1-E_{2}\right)}{E_{1}}+2 E_{3}\left(1+E_{2}\right)-2 E_{6}\left(1-E_{2}\right)\right. \\
&\left.\quad-2 E_{1} E_{7}-E_{1} E_{5}+E_{5}^{2}+4 E_{3} E_{4}-E_{1}-E_{5}\right) .
\end{aligned}
\end{aligned}
$$

The method basing on the use of general coefficients in series in perturbation of internal energy, was firstly used by Perelomova, Leble, KusmireK-OChrymiuk (2001) in regard to nonlinear propagation of sound in semi-ideal gases. The dynamic equation describing the progressive in the positive direction of axis $O X$ sound, follows from Eqs. (4), (7). More precisely, Eqs. (4) transform into three equivalent equations in view of relations (7), namely

$$
\begin{aligned}
& \frac{\partial \rho^{\prime}}{\partial t}+c_{0} \frac{\partial \rho^{\prime}}{\partial x}+\frac{1-D_{1}-D_{2}}{2} \frac{c_{0}}{\rho_{0}} \rho^{\prime} \frac{\partial \rho^{\prime}}{\partial x} \\
&+\frac{5+D_{1}^{2}+2 D_{1}\left(-3+D_{2}\right)+18 D_{2}-3 D_{2}^{2}-16 D_{3}-16 D_{4}-16 D_{5}}{32} \\
& \cdot \frac{c_{0}}{\rho_{0}^{2}} \rho^{\prime 2} \frac{\partial \rho^{\prime}}{\partial x}=0 .
\end{aligned}
$$

Equations (7), (9) (within accuracy up to the cubic terms) correspond to the famous Riemann wave existing in an ideal gas, the internal energy of which is well-known,

$$
e=\frac{p}{(\gamma-1) \rho}
$$

where $\gamma=C_{P} / C_{V}$ is the ratio of specific heats. The exact relations of perturbations in the Riemann wave are also well-known: 


$$
\begin{aligned}
& \left(1+\frac{\rho_{R}^{\prime}}{\rho_{0}}\right)=\left(1+\frac{\gamma-1}{2} \frac{v_{R}^{\prime}}{c_{0, i}}\right)^{2 / \gamma-1}, \\
& \left(1+\frac{p_{R}^{\prime}}{p_{0}}\right)=\left(1+\frac{\rho_{R}^{\prime}}{\rho_{0}}\right)^{\gamma}
\end{aligned}
$$

where $c_{0, i}=\sqrt{\gamma p_{0} / \rho_{0}}$ is an infinitely small signal sound speed in an ideal gas, and index $R$ marks quantities referring to the Riemann wave. Discarding terms of the order higher than three, one may rearrange Eqs. (11) into the following equalities:

$$
\begin{aligned}
\frac{v_{R}^{\prime}}{c_{0, i}} & =\frac{\rho_{R}^{\prime}}{\rho_{0}}+\frac{\gamma-3}{4}\left(\frac{\rho_{R}^{\prime}}{\rho_{0}}\right)^{2}+\frac{(\gamma-3)(\gamma-5)}{24}\left(\frac{\rho_{R}^{\prime}}{\rho_{0}}\right)^{3}, \\
\frac{p_{R}^{\prime}}{c_{0, i}^{2} \rho_{0}} & =\frac{\rho_{R}^{\prime}}{\rho_{0}}+\frac{\gamma-1}{2}\left(\frac{\rho_{R}^{\prime}}{\rho_{0}}\right)^{2}+\frac{(\gamma-1)(\gamma-2)}{6}\left(\frac{\rho_{R}^{\prime}}{\rho_{0}}\right)^{3} .
\end{aligned}
$$

It is easy to establish an accordance of Eqs. (7) and (12) in the case of an ideal gas, evaluating the constants in an ideal gas in appliance with Eqs. (5), (8), (10):

$$
\begin{aligned}
& E_{1}=-E_{2}=E_{4}=-E_{5}=-E_{7}=E_{9}=\frac{1}{\gamma-1}, \\
& E_{3}=E_{6}=E_{8}=D_{2}=\ldots=D_{5}=0, \\
& D_{1}=-\gamma .
\end{aligned}
$$

In view of (13), one can establish also the correspondence of Eq. (9) to the dynamic equation describing an excess density in the Riemann wave (written with accuracy of cubic terms)

$$
\frac{\partial \rho_{R}^{\prime}}{\partial t}+c_{0, i} \frac{\partial \rho_{R}^{\prime}}{\partial x}+\frac{\gamma+1}{2} \frac{c_{0, i}}{\rho_{0}} \rho_{R}^{\prime} \frac{\partial \rho_{R}^{\prime}}{\partial x}+\frac{(\gamma+1)(\gamma-3)}{8} \frac{c_{0, i}}{\rho_{0}^{2}} \rho_{R}^{\prime 2} \frac{\partial \rho_{R}^{\prime}}{\partial x}=0 .
$$

The quantities $M_{1}, \ldots M_{6}$ establish not only the parameters of nonlinearity $B / A=$ $2 M_{5} / M_{4}, C / A=6 M_{6} / M_{4}$, specified by $\left.p^{\prime}\left(\rho^{\prime}\right)\right|_{s, 0}$, but also three coefficients in series $\left.v^{\prime}\left(\rho^{\prime}\right)\right|_{s, 0}$, forming the relations analogous to those in the Riemann wave. As for the ratios $B / A$ and $C / A$, they may be expressed in terms of coefficients in the series of internal energy in the following manner:

$$
\begin{aligned}
& \frac{B}{A}=-\left(1+D_{1}+D_{2}\right), \\
& \frac{C}{A}=\left(2+3 D_{1}+2 D_{2}-2 D_{3}-2 D_{4}-2 D_{5}+D_{1}^{2}-D_{1} D_{2}\right),
\end{aligned}
$$


that yield for an ideal gas the well-known expressions:

$$
\begin{aligned}
& \frac{B}{A}=(\gamma-1), \\
& \frac{C}{A}=(\gamma-1)(\gamma-2) .
\end{aligned}
$$

\section{Examples of theory application}

2.1. The sound speed and parameters of nonlinearity $B / A$ and $C / A$ in the mixture consisting of water and its vapor in the phase equilibrium

Equations (15) are useful when internal energy of a fluid as a function of equilibrium pressure and density is established, regardless of the method of its determination, experimental or analytical. The useful analytical formula which describes internal energy in the mixture consisting of the boiling water and its vapor, is based on a variety of experimental data (KUZNETSOv, 1981):

$$
e(p, \rho)=4.1868 \cdot 10^{3}\left(71326.1 p^{0.245}+6.82997 p^{7 / 8} / \rho\right),
$$

where pressure $p$ is measured in $\mathrm{Pa}$, density $\rho$ in $\mathrm{kg} / \mathrm{m}^{3}$, and internal energy $e$ in $\mathrm{J} / \mathrm{kg}$. It is emphasized by KuzNETSOV (1981), that accuracy of partial derivatives of internal energy (17) is about $2-5 \%$ in the interval $3 \mathrm{~Pa} \leq p \leq 2 \cdot 10^{7} \mathrm{~Pa}$. The caloric equation of state depends on the mass concentration of vapor in the mixture $\alpha$ by means of equality:

$$
\rho=\frac{1}{\frac{1-\alpha}{\rho_{1}}+\frac{\alpha}{\rho_{2}}},
$$

connecting individual densities of liquid water $\rho_{1}$ and its vapor, $\rho_{2}$. The calculations undertaken by the authors uses the series $(5)$, where $e(p, \rho)$ is determined by Eq. (17). The parameters of nonlinearity are evaluated in accordance to Eqs. (15). Variations in pressure and temperature of the mixture are connected by the Clapeyron equation (LANDAU, Lifshitz, 1987):

$$
\frac{d p}{d T}=\frac{\triangle H}{\mu T\left(1 / \rho_{2}-1 / \rho_{1}\right)},
$$

where $\triangle H=40657 \mathrm{~J} / \mathrm{mol}$ is the enthalpy of vaporization of water. The vapor density was calculated according to the equation of state for an ideal gas:

$$
\rho_{2}=\frac{p \mu}{R T},
$$

where $\mu=18.015 \cdot 10^{-3} \mathrm{~kg} / \mathrm{mol}$ is the molar mass of water, $R=8.314 \mathrm{~J} /(\mathrm{mol} \cdot \mathrm{K})$ is the universal gas constant. That somewhat reduces the accuracy of evaluations, because the water vapor is a real gas, but has a small effect on thermodynamic 
a) $c_{0}, \frac{m}{s}$

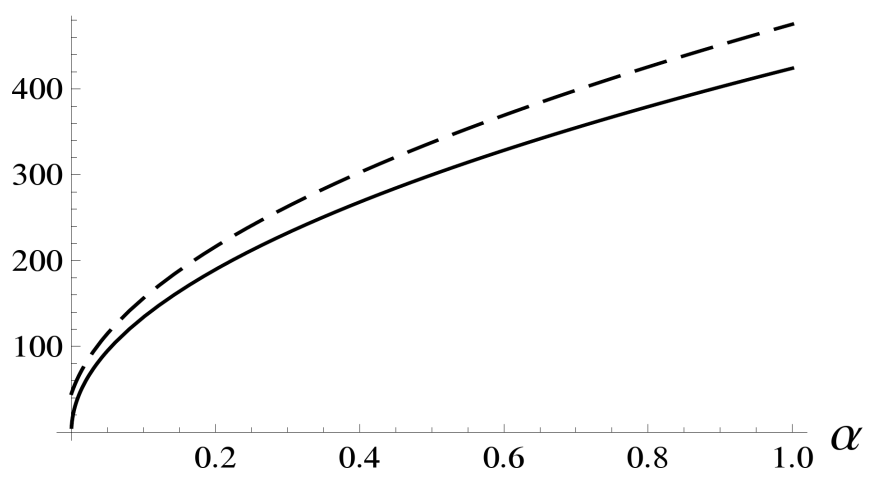

b) $\frac{B}{A}$

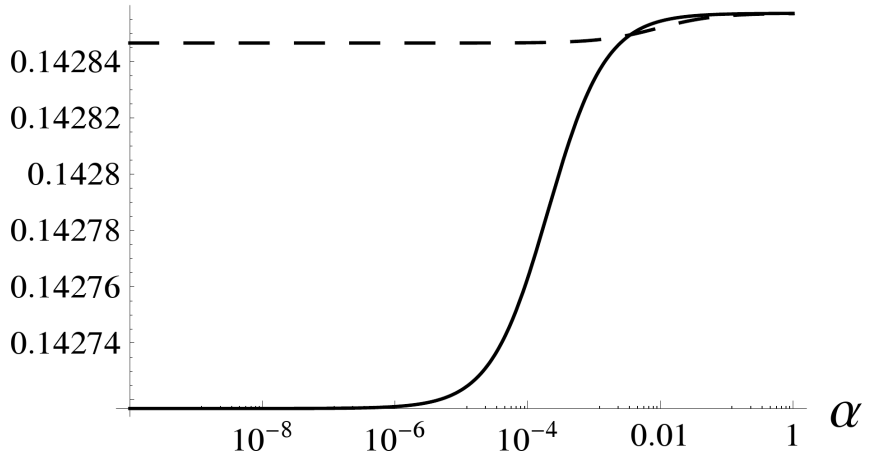

c) $-\frac{C}{A}$

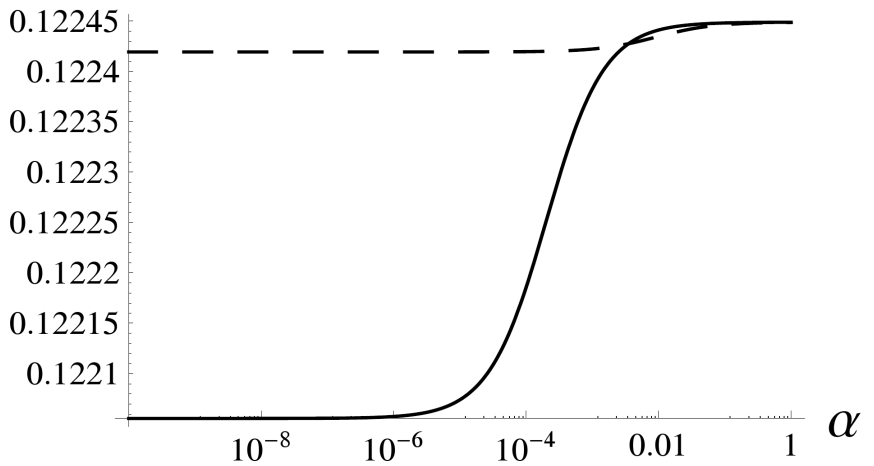

Fig. 1. The small-signal sound speed (a) and parameters of nonlinearity $B / A$ (b) and $-C / A$ (c) in the boiling mixture, consisting of liquid water and its vapor as functions of mass concentration of the vapor $\alpha$ at different temperatures of the mixture: $T_{0}=70^{\circ} \mathrm{C}$ (solid line), $T_{0}=200^{\circ} \mathrm{C}$ (dashed line). 
properties of the binary mixture as a whole. The authors have used the van der Waals equation of state for the water vapor in order to evaluate the sound velocity in the mixture and its parameters of nonlinearity. The results were very close to these of an ideal gas. It was proved by ARUTUNiAn (1994), that water vapor in the binary mixture may be considered as an ideal gas if pressure of the binary mixture is smaller than $1.3 \cdot 10^{7} \mathrm{~Pa}$. Data for the liquid water density were taken from the tables (Tables for water and its vapor - www pages [Ref. 27]). Figures 1a,b,c below show $c_{0}, B / A$ and $C / A$ as functions of mass concentration $\alpha$ at two different temperatures of the mixture in equilibrium state: $T_{0}=70^{\circ} \mathrm{C}$ (solid line) and $T_{0}=200^{\circ} \mathrm{C}$ (dashed line).

Sound velocity and parameter of nonlinearity $B / A$ of three-component systems with separate volumes of liquid, its vapor and a neutral gas were considered by one of the authors in the study (PERELOMOVA, 2005).

\subsection{Relations analogous to the Riemann wave in the van der Waals gases. Reflected wave in the van der Waals gases}

Consideration of gases obeying the van der Waals equation of state in the analytical form:

$$
\begin{aligned}
e & =\frac{1}{\gamma-1} \frac{R T}{\mu}-\frac{a \rho}{\mu^{2}}, \\
\frac{p}{\rho} & =\frac{R T}{\mu}\left(1+\frac{\rho}{\mu}\left(b-\frac{a}{R T}\right)\right),
\end{aligned}
$$

allows to determine not only the sound speed and ratios $B / A$ and $C / A$ (DENisov, $2002)$, but also the relations analogous to that in the Riemann wave ( $a, b$ are constants accounting for interaction between molecules and their volume, $\mu$ denotes a molar mass of a gas). The van der Waals gases internal energy as a function of pressure and density and the corresponding coefficients in the series of an excess internal energy, take the corrected form as follows:

$$
\begin{aligned}
e & =\frac{p}{\rho(\gamma-1)}-\frac{a \rho(\gamma-2)}{\mu^{2}(\gamma-1)}-\frac{b p}{\mu(\gamma-1)}, \\
E_{1} & =\frac{1}{\gamma-1}-\frac{b}{\mu \rho_{0}(\gamma-1)}, \\
E_{2} & =-\frac{1}{\gamma-1}-\frac{a \rho_{0}^{2}(\gamma-2)}{\mu^{2} p_{0}(\gamma-1)} .
\end{aligned}
$$

Equations (22) result not only in the corrected sound velocity and parameters of nonlinearity, that is the well-known point (Denisov, 2002), but also in the redetermined relations specific for the Riemann wave, according to Eqs. (5), (7), (8). The table below shows additional factors in the series for velocity and excess 


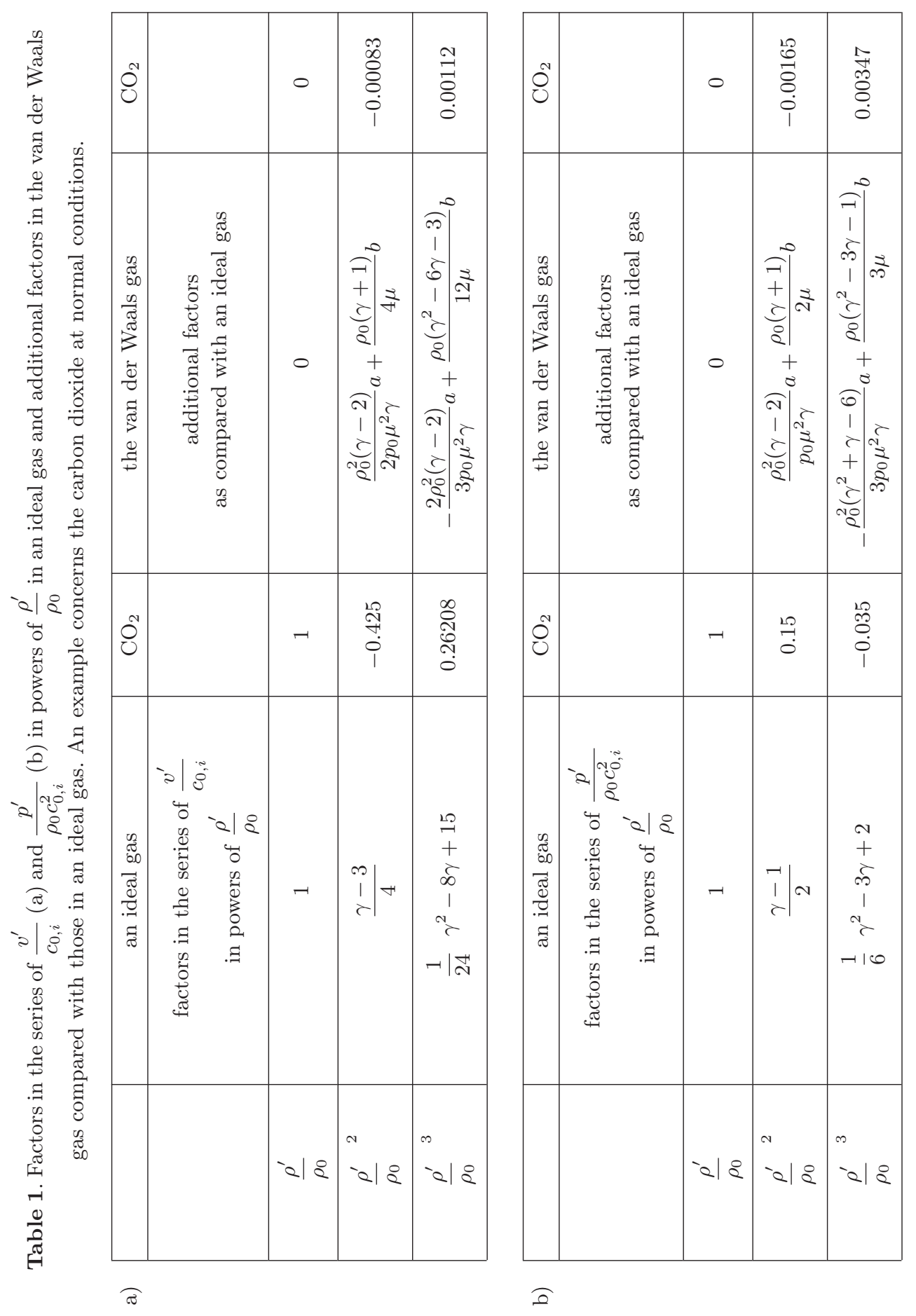


pressure as compared with that of an ideal gas, and the corresponding estimations for the carbon dioxide $\mathrm{CO}_{2}$ at the room temperature and atmospheric pressure.

The coefficients in the van der Waals equation for $\mathrm{CO}_{2}$ are

$$
a=0.364 \mathrm{~m}^{6} \mathrm{~Pa} / \mathrm{mol}^{2}, \quad b=0.0000427 \mathrm{~m}^{3} / \mathrm{mol}
$$

(Grigoryev, Meilikhov, 1991). The molar mass and adiabatic factor equal, respectively, $\mu=0.044 \mathrm{~kg} / \mathrm{mol}, \gamma=1.3$.

Correct determination of the factors in both Eqs. (7) is of importance in context of the reflected wave. This phenomena were observed during periodic in time, planar wave propagation, due to absorption of a primary wave independently of the absorption type (due to standard attenuation or nonlinear attenuation at the front of a shock wave) (MAKAROv, OCHMAnN, 1996). Averaging the continuity equation with respect to time, and taking into account the sound periodicity and absence of the mean mass flow on the transducer, one obtains a quantity of the mass flux averaged over acoustic period equal zero (bracketed quantities denote temporal averaging over the sound period):

$$
\left\langle\left(\rho_{0}+\rho^{\prime}\right) v^{\prime}\right\rangle=0
$$

so that $\left\langle v^{\prime}\right\rangle=-\left\langle v^{\prime 2}\right\rangle / c_{0, i}$, and, therefore, from Eqs. (12) and (23) it follows, that

$$
\left\langle\rho^{\prime}\right\rangle=-(\gamma+1) \rho_{0}\left\langle v^{\prime 2}\right\rangle /\left(4 c_{0, i}^{2}\right)
$$

and

$$
\left\langle p^{\prime}\right\rangle=(\gamma-3) \rho_{0}\left\langle v^{\prime 2}\right\rangle / 4
$$

in an ideal gas. On the other hand, the total time-averaged momentum flux should be a constant through over the whole spatial domain, but is not actually,

$$
\Pi=\left\langle p^{\prime}+\rho_{0} v^{\prime 2}\right\rangle=\frac{\rho_{0}(\gamma+1)}{4}\left\langle v^{\prime 2}\right\rangle \neq \text { const. }
$$

Hence, relations in the Riemann rightwards progressive wave do not guarantee zero time-averaged momentum flux, as was proved by MAKAROV, OCHMANN (1996). This last value is not constant in an absorbing flow, independently of the mechanism of absorption. It follows from the above reasoning a well-known conclusion that the attenuated sound field under closed vessel conditions cannot be simulated by the sound progressive in one direction, because it does not hold constant time-averaged momentum flow. Since a convective flow (streaming) does not exist in the one-dimensional geometry, the only possibility to avoid this contradiction is to consider a self-reflected wave traveling to the left and having the pressure

$$
p_{\text {refl }}^{\prime}=-\frac{\rho_{0}(\gamma+1)}{8}\left\langle v^{\prime 2}\right\rangle .
$$

This pressure wave being the second-order quantity, provides a proper constant value of a summary momentum flow (zero if a primary wave propagates in infinitely long tube and its amplitude tends to zero at $x \rightarrow \infty$ ). Actually, the 
self-reflection as an original object of study was first introduced by RUDENKO et al. (1970) and then discussed in some details in the book (RUdEnKo, SoluYAN 1977). Later, MAKAROV (1994) has developed the theory of reflected wave in the absorbing flow, not necessarily one-dimensional. MAKAROV (1994) explains the appearance of the self-reflected wave by changes in the Riemann invariant caused by absorption. These small perturbations propagate along negative characteristics.

The reflected wave in the van der Waals gas differs from that in an ideal gas. Application of the corrected relations compared with that in the Riemann wave from Table 1, and evaluation of the excess pressure in the reflected wave yield:

$$
p_{\text {refl }}^{\prime}=-\left(\frac{\rho_{0}(\gamma+1)}{8}-\frac{\rho_{0}^{2}(\gamma-2)}{4 p_{0} \mu^{2} \gamma} a-\frac{\rho_{0}(\gamma+1)}{8 \mu} b\right)\left\langle v^{\prime 2}\right\rangle
$$

Large values of the self-reflected wave pressure are hardly expected to be produced by a single pulse, but total pressure may be significant as a sum of waves reflected from many periods of sound (MAKArov, OChMANn, 1996; MAKAROV, 1994). The experimental studies of the reflected wave were already started (NAKAMURA, NAKASHIMA, 1987). The higher-order nonlinear corrections to an excess pressure of the reflected wave may be derived by involving of the higher-order terms in the both relations, analogous to that of the Riemann wave (Eq. (7)). The reflected wave excess pressure corrected by cubic terms takes the form

$$
\begin{aligned}
p_{\text {refl }}^{\prime}= & \frac{\rho_{0}\left(D_{1}+D_{2}-1\right)}{8}\left\langle v^{\prime 2}\right\rangle \\
& +\frac{\rho_{0}}{48 c_{0}}\left(1-4 D_{1}-2 D_{2}+4 D_{3}+D_{4}+4 D_{5}+3 D_{1}^{2}+5 D_{2}^{2}+8 D_{1} D_{2}\right)\left\langle v^{\prime 3}\right\rangle .
\end{aligned}
$$

Equation (27) may be easily rearranged into the reflected wave excess pressure in a flow over an ideal gas:

$$
p_{\text {refl }}^{\prime}=-\frac{\rho_{0}(\gamma+1)}{8}\left\langle v^{\prime 2}\right\rangle+\frac{\rho_{0}(\gamma+1)(3 \gamma+1)}{48 c_{0, i}}\left\langle v^{\prime 3}\right\rangle .
$$

The conclusion about negative excess pressure of the reflected wave remains valid in the case of a weak nonlinearity, when acoustic Mach number is small:

$$
M=\frac{v^{\prime}}{c_{0}} \ll 1 \text {. }
$$

\section{Conclusions}

The sound features, its speed and nonlinear distortion during propagation in fluids, may be measured experimentally. While the small-signal sound speed is easy to measure, it is difficult to measure the parameters of nonlinearity. As a rule, a fairly detailed information about dependence of the sound speed on pressure and temperature, $c(p, T)$, is required (see Eq. (3)) for determination 
of the ratios $B / A$ and $C / A$. The determination of $C / A$ requires especially precise knowledge of curvature of the surface $c(p, T)$, what is difficult to obtain within reasonable accuracy. Along with experimental studies, the conclusions of chemical physics are very useful. Producing analytical formulae for thermodynamic functions with a high accuracy, they in fact allow to evaluate the sound speed and both parameters of nonlinearity in a wide domain of thermodynamic equilibrium parameters, without complex experiments. On the other hand, the conclusions of chemical physics are based on experimental thermodynamic data and the general concept of interactions of molecules. Thermodynamic functions for some important substances (even empirically determined), in spite of the fact they have been obtained in non-acoustic purposes, are very useful for evaluation of sound features.

The analytical formulae for the sound speed and parameters of nonlinearity derived in this study, are applied in studies of two examples of fluids different from an ideal gas, namely water being in the phase equilibrium with its vapor, and the van der Waals gases. Some theoretical predictions concerning parameters of nonlinearity in the binary mixture may be drawn out: $B / A$ increases, and $C / A$ decreases with increase of vapor mass concentration $\alpha$. Both ratios, along with the sound speed, depend on temperature of the mixture in accordance to Fig. 1. The analysis of the van der Waals gases results, among other, in the relations analogous to that in the Riemann wave and to an excess pressure in the reflected wave.

During the 1990s and 2000s, a wealth of applications of nonlinearity have been developed, among other nonlinear absorption of the high-intensity wave, nonlinear sound wave interactions, parametric transmitting and receiving, acoustic heating and streaming (nonlinear generation of the entropy and vorticity modes, correspondingly) (Rudenko, Soluyan, 1977; Makarov, Ochmann, 1996; TјøтtA, Тлøтta, 1993; Leighton, 2007; Naugolnykh, 2008). Biomedical applications are very important, such as medical imaging, high-intensity focused ultrasound and pulse propagation, also over an inhomogeneous fluid and tissue (CARstensen, BaCon, 1998; Leighton, 2007; NAugolnykh, 2008; Duck et al., 1998; Kennedy, Cranston, 2003). The most quickly developing and promising area of scientific interest is medical application of ultrasound (including therapeutic) (CARstensen, BACON, 1998; Leighton, 2007; DuCK et al., 1998; Kennedy, Cranston, 2003). Thermodynamics of biological tissues, first of all, caloric equation of state, differs essentially from an ideal gas. Fortunately, the encouraging achievements of physical chemistry allow determination of analytical thermodynamic equations of state in many fluids within reasonable accuracy. The correct description of nonlinear effects both in sound itself and in the secondary non-acoustic phenomena caused by it, heating, streaming and cavitation, are of great importance in medicine and technique (Leighton, 2007; NAUGOLNYKH, 2008). Both non-acoustic modes and the reflected wave caused by absorption in a highly viscous fluid (like human blood) may distort the readings of the instru- 
ments and must be correctly recognized. The conclusions of this study may be useful in evaluation of nonlinear effects of sound in biological tissues and other fluids with caloric equation of state different from that in ideal gases.

\section{References}

1. Arutunian G.M. (1994), Thermodynamical theory of heterogeneous systems [in Russian], Fizmatlit, Moscow.

2. Beyer R.T. (1960), Parameter of nonlinearity in fluids, J. Acoust. Soc. Am., 32, 6, 719721.

3. Beyer R.T. (1998), The parameter B/A, [in:] Nonlinear Acoustics, Hamilton M.F., Blackstock D.T. [Eds.], pp. 25-39, Academic Press, New York.

4. Carstensen E.L., Bacon D.R. (1998), Biomedical applications, [in:] Nonlinear Acoustics, Hamilton M.F., Blackstock D.T. [Eds.], pp. 421-447, Academic Press, New York.

5. Coppens A.B., et al. (1965), Parameter of nonlinearity in fluids II, J. Acoust. Soc. Am., 38, 5, 797-804.

6. Denisov D.A. (2002), Calculation of the nonlinearity parameter for some types of gaseous and liquid mixtures, Acoustical Physics, 48, 4, 430-435.

7. Duck F.A., Baker A.C., Starrit H.C. (1998), Ultrasound in medicine, IOP Publishing Ltd, Bristol and Philadelphia.

8. Fox F.E., Wallace W.A. (1954), Absorption of finite amplitude sound waves, J. Acoust. Soc. Am., 26, 994-1006.

9. Grigoryev I.S., Meilikhov Ye.Z. [Eds.], (1991), Physical values [in Russian], Energoatomizdat, Moscow.

10. Jeffery C.A., Austin P.H. (1999), A new analytic equation of state for liquid water, J. Chem. Phys., 110, 1, 484-496.

11. Kennedy J.E., Cranston D. (2003), High intensity focused ultrasound: surgery of the future?, British Journal of Radiology, 76, 590-599.

12. Kuznetsov M.M. (1981), Two-phase mixture consisting of liquid water and its vapor. Equation of state, velocity of sound, isentropies, Doklady AN SSSR 257, 858-860.

13. Landau L.D., Lifshitz E.M. (1987), Fluid Mechanics, 2nd Ed., 6, Butterworth-Heinemann, UK, Oxford.

14. Lee J.F., Sears F.W., Turcotte D.L. (1963), Statistical thermodynamics, AddisonWesley Publishing Company, London.

15. Leighton T.G. (2007), What is ultrasound?, Progress in Biophysics and Molecular Biology, 93, 1-3, 3-83.

16. MakARov S. (1994), Self-reflection in nonlinear acoustics. Theoretical ground and possible applications, Acustica, 80, 1-13.

17. Makarov S., Ochmann M. (1996), Nonlinear and thermoviscous phenomena in acoustics, Part I, Acustica, 82, 579-606. 
18. Nakamura, Y., Nakashima Y. (1987), Analysis of unsteady shock wave propagation generated by interaction with simple expansion wave, Techn. Reps. Kumamoto Univ., 36, $1,31-40$.

19. Naugolnykh K. (2008), 50 years of nonlinear acoustics, Nonlinear Acoustics - fundamentals and applications, 18th International Symposium on Nonlinear Acoustics, EnflO B.E., Hedberg C.M., Kari L. [Eds.], pp. 3-8, AIP Conference Proceedings, Melville, New York.

20. Novikov B.K., Rudenko O.V., Timoshenko V.I. (1988), Nonlinear underwater acoustics, J. Acoust. Soc. Am., 84, 1, 458-459.

21. Perelomova A. (2005), Parameter of nonlinearity B/A of three-component systems with separate volumes of liquid, its vapor and a neutral gas, Archives of Acoustics, 30, 3, 333343.

22. Perelomova A., Leble S., Kusmirek-Ochrymiuk M. (2001), Nonlinear evolution of the acoustic wave in a semi-ideal gas, Archives of Acoustics, 26, 4, 351-360.

23. Riemann B. (1953), The Collected Works of Bernard Riemann, pp. 156-175, Dover, New York.

24. Rudenko O.V., Soluyan S.I. (1977), Theoretical foundations of nonlinear acoustics, Plenum, New York.

25. Rudenko O.V., Soluyan S.I., Khokhlov R.V. (1970), Formation of reflected waves at discontinuities in a sound wave, Sov. Phys. Acoust., 15, 355-359.

26. Sehgal C.M. (1995), Nonlinear ultrasonics to determine molecular properties of pure liquids, Ultrasonics, 33, 2, 155-161.

27. Tables for water and its vapor: http://wipos.p.lodz.pl/baza/spis04.html

28. Тлøтта S., Тృøтта J.N. (1993), Acoustic streaming in ultrasonic beams, Advances in Nonlinear Acoustics, Proceedings of the 13th International Symposium on Nonlinear Acoustics, Hobaek H. [Ed.], pp. 601-606, World Scientific, Singapore. 